\title{
Loss of NR1 Subunit of NMDARs in Primary Sensory Neurons Leads to Hyperexcitability and Pain Hypersensitivity: Involvement of $\mathrm{Ca}^{2+}$-Activated Small Conductance Potassium Channels
}

\author{
Promila Pagadala, ${ }^{1 *}$ Chul-Kyu Park, ${ }^{2 *}$ Sangsu Bang, ${ }^{2 *}$ Zheng-Zhong Xu, ${ }^{2}$ Rou-Gang Xie, ${ }^{2}$ Tong Liu, ${ }^{2}$ Bao-Xia Han, ${ }^{1}$ \\ W. Daniel Tracey Jr, ${ }^{1,2}$ Fan Wang, ${ }^{1}$ and Ru-Rong Ji ${ }^{2}$ \\ ${ }^{1}$ Departments of Cell Biology and Neurobiology and ${ }^{2}$ Departments of Anesthesiology and Neurobiology, Duke University Medical Center, Durham, North \\ Carolina 27710
}

It is well established that activation of NMDARs plays an essential role in spinal cord synaptic plasticity (i.e., central sensitization) and pain hypersensitivity after tissue injury. Despite prominent expression of NMDARs in DRG primary sensory neurons, the unique role of peripheral NMDARs in regulating intrinsic neuronal excitability and pain sensitivity is not well understood, in part due to the lack of selective molecular tools. To address this problem, we used Advillin-Cre driver to delete the NR1 subunit of NMDARs selectively in DRG neurons. In NR1 conditional knock-out (NR1-cK0) mice, NR1 expression is absent in DRG neurons but remains normal in spinal cord neurons; NMDA-induced currents are also eliminated in DRG neurons of these mice. Surprisingly, NR1-cKO mice displayed mechanical and thermal hypersensitivity compared with wild-type littermates. NR1-deficient DRG neurons show increased excitability, as indicated by increased frequency of action potentials, and enhanced excitatory synaptic transmission in spinal cord slices, as indicated by increased frequency of miniature EPSCs. This hyperexcitability can be reproduced by the NMDAR antagonist APV and by Ca ${ }^{2+}$-activated slow conductance $\mathrm{K}^{+}$(SK) channel blocker apamin. Furthermore, NR1-positive DRG neurons coexpress SK1/SK2 and apamin-sensitive afterhyperpolarization currents are elevated by NMDA and suppressed by APV in these neurons. Our findings reveal the hitherto unsuspected role of NMDARs in controlling the intrinsic excitability of primary sensory neurons possibly via Ca ${ }^{2+}$-activated SK channels. Our results also call attention to potential opposing effects of NMDAR antagonists as a treatment for pain and other neurological disorders.

\section{Introduction}

It is well established that NMDARs expressed centrally in secondorder nociceptive neurons in the spinal cord and medullary dorsal horn play an essential roles in the generation of central sensitization and pain hypersensitivity after tissue injury (Ji et al., 2003). However, their role in primary sensory neurons remains unclear. NMDARs, including NR1 and NR2 subunits, are expressed in DRG neurons (Sato et al., 1993). NMDARs synthe-

Received Jan. 30, 2013; revised June 21, 2013; accepted July 15, 2013.

Author contributions:P.P., C.-K.P., S.B., Z.-Z.X., T.L., F.W., and R.-R.J. designed research;P.P.,C.-K.P., S.B., Z.-Z.X., R.-G.X., T.L., and B.-X.H. performed research; P.P., C.-K.P., S.B., Z.-Z.X., R.-G.X., T.L., and B.-X.H. analyzed data; W.D.T., F.W., and R.-R.J. wrote the paper.

The work was supported by the National Institutes of Health (Grants \#R01DE19440 and \#R01DE19440S1 to F.W., Grants \#R01DE17794, \#R01DE22743, and \#R01NS67686 to R.R.J., Grants \#R01NS054899 and \#R01GM086458 to W.D.T., and Grant \#R21NS082985 to Z.Z.X.).

The authors declare no competing financial interests.

*P.P., C.-K.P., and S.B. contributed equally to this work.

Correspondence should be addressed to either of the following: Ru-Rong Ji, Departments of Anesthesiology and Neurobiology, Duke University Medical Center, Box DUMC 3094, Durham, NC 27010, E-mail: ru-rong.j@duke.edu; or Fan Wang, Departments of Cell Biology and Neurobiology, Duke University Medical Center, Box 3709, Durham, NC 27010,E-mail: fan.wang@dm.duke.edu.

DOI:10.1523/JNEUROSCI.0454-13.2013

Copyright $\odot 2013$ the authors $\quad 0270-6474 / 13 / 3313425-06 \$ 15.00 / 0$ sized in DRG neuronal somata can be transported to peripheral terminals in skin and muscle and central terminals in the spinal cord and brainstem trigeminal nucleus (Liu et al., 1997; Coggeshall and Carlton, 1998). Both NMDARs in peripheral and central terminals of primary afferent neurons have been implicated in nociception, but the reports were contradictory (Liu et al., 1997; Bardoni et al., 2004). Peripheral glutamate release and activation of NMDARs caused pain hypersensitivity after inflammation (deGroot et al., 2000; Du et al., 2003). Several studies further demonstrated that peripheral administration of NMDAR antagonists or genetic deletion of NR1 subunit in subset of primary sensory neurons inhibited pain in rodents (Du et al., 2003; McRoberts et al., 2011). Topical injection of the NMDAR antagonist ketamine also reduced allodynia in patients with complex regional pain syndrome (Finch et al., 2009). In spinal cord central afferents, activation of NMDARs by NMDA application released the neuropeptide substance P to elicit pain (Liu et al., 1997). Conversely, studies also showed that peripheral application of the NMDAR antagonist MK-801 or ketamine had essentially no effects in reducing inflammatory and neuropathic pain (Aley and Levine, 2002; Sawynok and Reid, 2002). Furthermore, activation of spinal cord presynaptic NMDARs by exogenous glutamate 
inhibited glutamate release from the sensory terminals (Bardoni et al., 2004), suggesting an antinociceptive role of presynaptic NMDARs. Therefore, whether the role of NMDARs in primary sensory neurons is pronociceptive or antinociceptive remains an unresolved issue.

To determine the physiological role of peripheral NMDARs, we specifically deleted NR1, the essential subunit of NMDARs, in DRG sensory neurons by crossing NR1 $1^{\text {flox/flox }}$ mice with the sensory-specific Cre line Advillin ${ }^{\mathrm{Cre} /+}$ (Zhou et al., 2010; da Silva et al., 2011), hereafter referred to as NR1-cKO mice. Unexpectedly, we found hyperexcitability in DRG neurons of these NR1cKO mice due to possible suppression of $\mathrm{Ca}^{2+}$-activated small conductance $\mathrm{K}^{+}$(SK) channels.

\section{Materials and Methods}

Mice. NR $1^{\text {flox/flox }}$ mice were from The Jackson Laboratory (JAX stock \#005246) and genotyping was performed as described previously (McHugh et al., 2007). Advillin ${ }^{\mathrm{Cre} /+}$ mice were described previously (Zhou et al., 2010; da Silva et al., 2011). Male Advillin ${ }^{\text {Cre/+ }}$ mice were used to cross to female NR1 $1^{\text {flox/flox }}$ mice first to obtain male Advillin ${ }^{\mathrm{Cre} /+} ; \mathrm{NR} 1^{\text {flox/++}}$ mice, which were crossed again to female NR $1^{\text {flox } / \text { flox }}$ mice to generate Advillin ${ }^{\mathrm{Cre} /+} ; \mathrm{NR} 1^{\text {flox/flox }}(\mathrm{NR} 1-\mathrm{cKO})$ mice. Adult mice (8-12 weeks of age, both males and females, with sex and age matched) were used for behavioral studies. Acute inflammatory pain was induced by injection of formalin $(5 \%, 20 \mu \mathrm{l}$, Sigma) into a hindpaw. Young mice (4-6 weeks old) were used for electrophysiological studies. All animal procedures were approved by the animal care committee of Duke University.

Histology. The cDNA fragment of NR (McHugh et al., 2007) was amplified by PCR and used as template for synthesizing antisense probe. In situ hybridization was performed according to standard procedures (Hasegawa et al., 2007). For immunofluorescence, anti-CGRP and anti-vGlut1 (1:1000; Millipore) primary antibodies and Alexa Fluor 488- or Alexa Fluor 568-conjugated secondary antibodies (1:5000; Invitrogen) were used. IB4 staining was performed with FITC-IB4 (1:2000; Invitrogen).

Whole-cell patch-clamp recordings in dissociated DRG neurons. As described previously (Park et al., 2011), DRGs were digested with collagenase/dispase II, followed by trypsin, and mechanically dissociated. DRG cells were grown in a neurobasal defined medium for $24 \mathrm{~h}$ before use. Whole-cell voltage-clamp and current-clamp recordings were performed at room temperature to measure currents and action potentials, respectively (Liu et al., 2012) using an Axopatch-200B amplifier (Molecular Devices). The resistance of the pipettes was 4-5 M 2 . NMDAinduced currents were recorded under voltage clamp $\left(V_{\mathrm{m}}=-60 \mathrm{mV}\right)$. Action potentials were recorded under current clamp with $1 \mathrm{~s}$ depolarizing current pulses of $100 \mathrm{pA}$ amplitude.

Apamin-sensitive outward tail currents (afterhyperpolarization [AHP] currents) were recorded as described previously with some modifications (Gold et al., 1996) and validated by the selective SK channel blocker apamin (100 nm; Sigma). Briefly, SK currents were isolated under voltage clamp with a $400 \mathrm{~ms}$ depolarizing pulse (from -60 to $0 \mathrm{mV}$, holding at $-60 \mathrm{mV}$ ). To define NMDA-dependent SK currents, a second pulse was given (from -80 to $0 \mathrm{mV}$, holding at $-60 \mathrm{mV}$ ). The duration between the two pulses was $900 \mathrm{~ms}$. The pipette solution for recording action potentials and SK currents contains contained the following (in $\mathrm{mm}$ ): $126 \mathrm{~K}$-gluconate, $10 \mathrm{NaCl}, 1 \mathrm{MgCl}_{2}, 10 \mathrm{EGTA}, 2 \mathrm{NaATP}$, and 0.1 MgGTP, adjusted to $\mathrm{pH} 7.4$ with $\mathrm{KOH}$ and osmolarity 295-300 mOsm. Some DRG neurons were also pretreated with APV (50 $\mu \mathrm{M}$; Sigma) for $10 \mathrm{~min}$.

Spinal cord slice preparation and patch-clamp recordings. The L4-L5 lumbar spinal cord segment was removed and transverse slices $(400 \mu \mathrm{m})$ were prepared and perfused with Kreb's solution at $36 \pm 1^{\circ} \mathrm{C}$. The wholecell recordings were made from lamina IIo neurons in voltage-clamp mode (Park et al., 2011). Neurons were held at the potential of $-70 \mathrm{mV}$ to record miniature EPSCs in the presence of $1 \mu \mathrm{M}$ TTX. The resistance of a typical patch pipette was 5-10 M $\Omega$. Data were analyzed with Mini Analysis software (Synaptosoft).

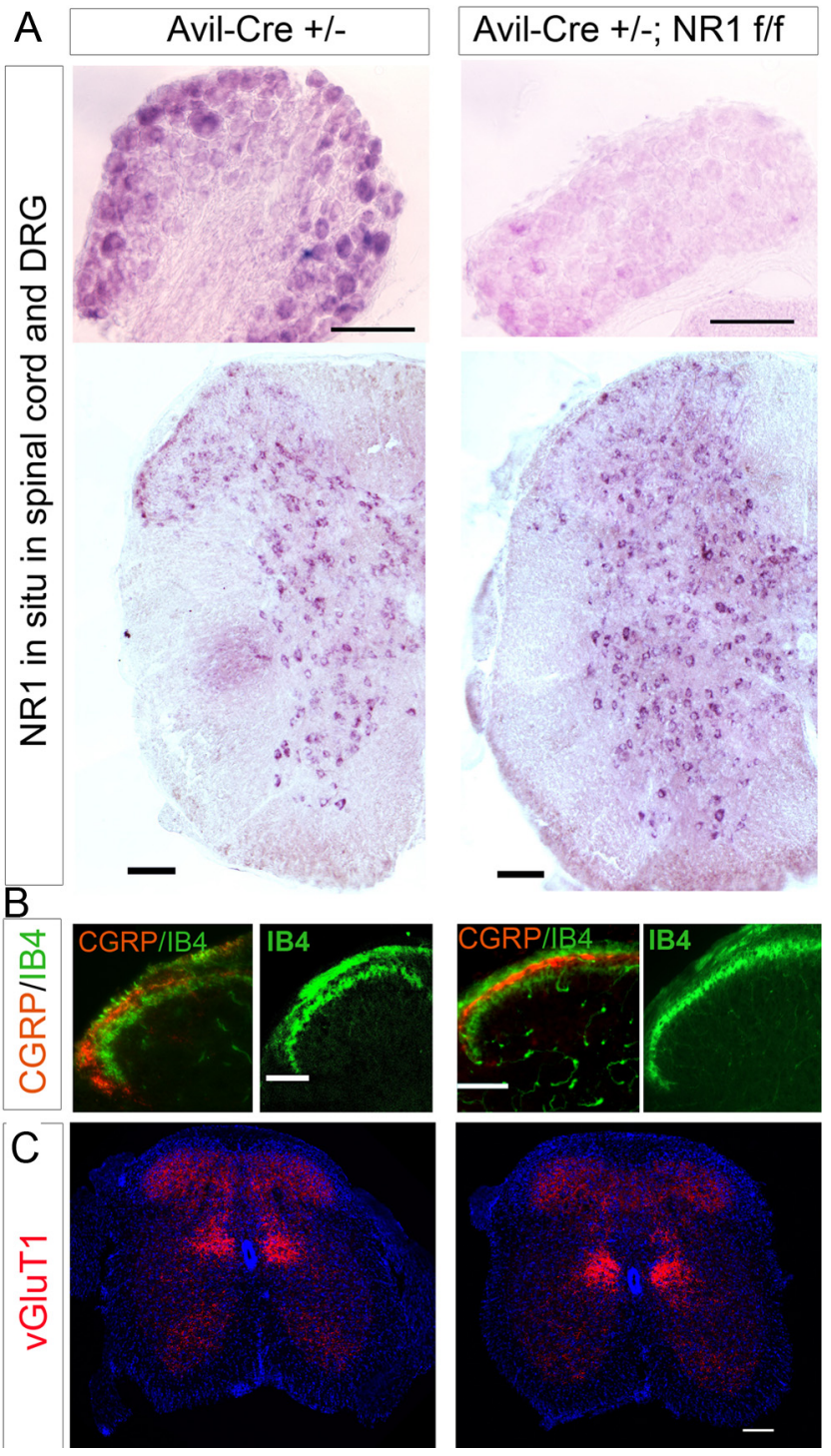

Figure 1. NR1 subunit is specifically deleted in the DRG sensory neurons of the NR1-cKO mice. $\boldsymbol{A}$, Representative images of NR1 in situ hybridization results on DRG and spinal cord sections from either Advillin-Cre; NR1-wild-type mice (left) or Advillin-Cre; NR1 flox/flox (NR1cKO) mice (right). NR1 mRNA is selectively deleted in DRG neurons in NR1-cKO mice. $\boldsymbol{B}$, Representative images of anti-CGRP/IB4 double staining or IB4 staining alone on spinal cord sections from NR1-wild-type (left) or NR1-cKO (right) mice. C, Representative images of anti-vGluT1 staining on spinal cord sections from NR1-wild-type (left) or NR1-cK0 (right) mice. The IB4 nonspecific staining in deep layers stains blood vessels. Scale bars, $100 \mu \mathrm{m}$.

Whole-cell patch-clamp recordings in whole-mount DRG. The L4-L5 DRGs were removed and placed in cold oxygenated ACSF. DRGs were digested with a mixture of $1.0 \mathrm{mg} / \mathrm{ml}$ protease and $1.6 \mathrm{mg} / \mathrm{ml}$ collagenase (Sigma) for $30 \mathrm{~min}$ at $37^{\circ} \mathrm{C}$ and transferred into a holding chamber containing normal $\mathrm{Mg}^{2+}$-free ACSF with CNQX $(2 \mu \mathrm{M})$ at $26^{\circ} \mathrm{C}$. Neurons were hold at $-60 \mathrm{mV}$ for voltage-clamp recordings. Inward current was inducted after continuous current injection (20-30 times) to induce artificial action potentials.

Single-cell RT-PCR. A single neuron was aspirated into a patch pipette, kept in a reaction tube containing reverse transcription reagents, and incubated for $1 \mathrm{~h}$ at $50^{\circ} \mathrm{C}$. The cDNA product was then used in separate PCR. The first and second round PCR was performed using "outer" and "inner" primers, respectively. A negative control was obtained from pipettes that did not harvest any cell contents (Liu et al., 2012). The sequences of all primers were as follows: SK1 (outer): $5^{\prime}-A A G A C G C T C A$ TGACCATCTGC-3', 5'-GTGTCCATCATGAAGTTGTGCA-3'; SK1 (inner): 5' -CTGTGTTGTTGGTCTTCAGCGT-3', 5'-CAGTGAGCAGA 
A Voltage-clamp recordings
WT

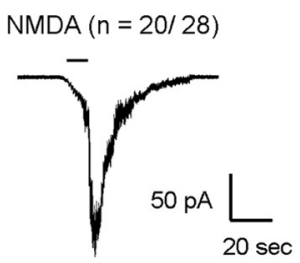

NR1-cKO
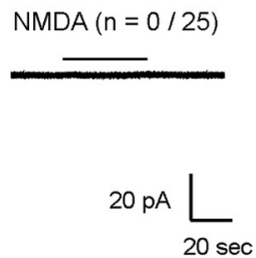

B

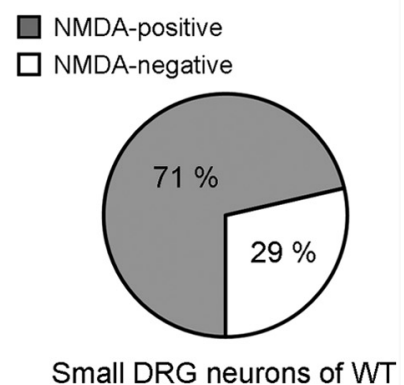

C Current-clamp recordings

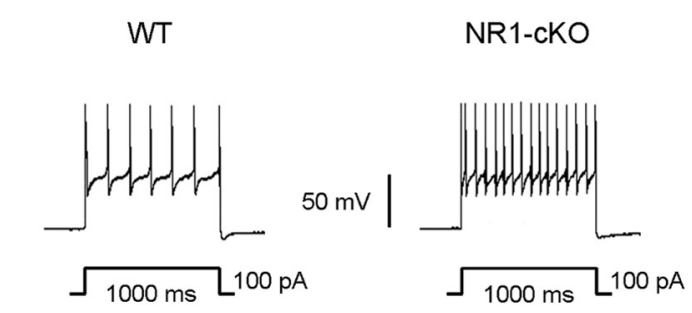

E

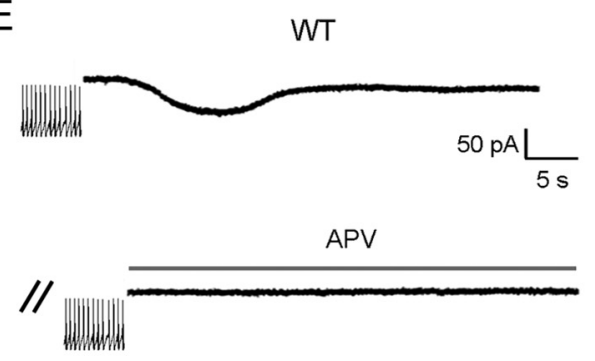

$\mathrm{F}$

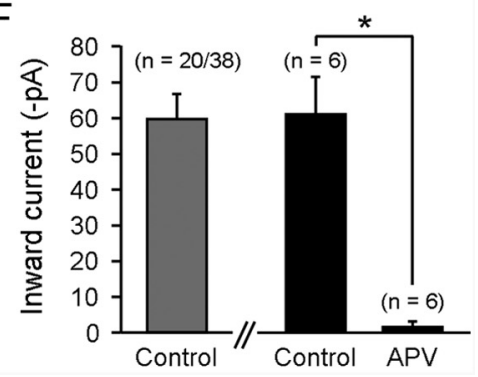

Figure 2. NR1-CKO mice display increased frequency in action potentials in small-sized DRG neurons. $A$, NMDA (500 $\mu \mathrm{M})$ induces inward current in WT neurons (20/28 neurons), but not in NR1-deficient neurons ( $n=0 / 25$ neurons). $\boldsymbol{B}$, Venn diagram showing portion of small DRG neurons responding to NMDA. $C$, Traces of single and repetitive action potentials induced by current injection in WT and NR1-deficient neurons ( $n=15$ neurons/group). $\boldsymbol{D}$, Action potential frequencies in WT and NR1-deficient neurons after current injection $(25,100$, and $250 \mathrm{pA}) .{ }^{*} p<0.05$ compared with WT, $n=8$ neurons/group. $\boldsymbol{E}, \boldsymbol{F}$, Artificial action potentials induce inward current in a neuron in whole-mount DRG, which is blocked by APV $(50 \mu \mathrm{m})$. $\boldsymbol{F}$, Amplitude of the inward currents recorded in whole-mount DRG after action potentials. ${ }^{*} p<0.05$, paired $t$ test, $n=20$ or 6 neurons per group.

(two groups) or one-way ANOVA (multiple groups) followed by post hoc Bonferroni test. The criterion for statistical significance was $p<0.05$.

\section{Results}

Sensory-neuron-specific deletion of NR1 subunit

We first examined NR1 expression in the DRG and spinal cord of NR1-cKO and wild-type littermate control mice carrying only the Advillin ${ }^{\mathrm{Cre} /+}$ allele. In situ hybridization revealed that NR1 mRNA is expressed in most DRG neurons of both small and large sizes of control mice but is absent in almost all DRG neurons of NR1cKO mice (Fig. 1A). NR1 mRNA is also expressed in a large number of neurons in spinal cord in both control and cKO mice and no difference was noticed between the genotypes (Fig. 1A). These data confirmed a selective deletion of NR1 mRNA in DRG neurons.

Next, we examined the axonal projections of distinct groups of sensory neurons to the spinal cord using anti-CGRP (marker for peptidergic nociceptive neurons), FITC-IB4 (a marker for nonpeptidergic nociceptive neurons), and anti-vGluT1 (a marker for A-fiber touch and proprioceptive neurons). NMDARdeficient DRG sensory neurons showed grossly normal layered patterns of projections into the spinal cord: CGRP/IB4 projections in the superficial dorsal horn (laminae I-II) and vGluT1 projections in the deep dorsal horn (laminae III-IV) (Figs. $1 B, C$ ). Our data suggest that NR1-cKO mice do not have noticeable developmental defects in DRG and spinal cord.

CACACACCCTT-3'; SK2 (outer): 5'-ATGGATAATTGCCGCATGGA3', 5'-TTCTTGCCACTACGGCTACCAC-3'; SK2 (inner): 5'-TCCGAG CTTGTGAAAGGTACCA-3', 5' -TGCAACCTGCACCCATGATT-3'; SK3 (outer): 5' -TGTCAGGCTGCGTGAATAGTAA-3', 5' -TTTGTGCT TTTCCTCCACCAG-3' ${ }^{\prime}$;SK3(inner):5' -CCTGCCGGACAAGGAATATG T-3', TGCTCGCAGCTTCCAGAATA-3'; NR1 (outer): 5'-GCTTCATG GAAGACCTGGATAA-3', 5'-GTGGAGGTGATAGCCCTAAATG-3'; NR1(inner):5'-TGACTCCCGCAGCAATG-3', 5' -TTTCTATCCTGCAG GTTCTTCC-3'; GAPDH (outer): 5' -AGCCTCGTCCCGTAGACAAAA3', $5^{\prime}$-TTTTGGCTCCACCCCTTCA-3'; GAPDH (inner): 5' -TGAAGG TCGGTGTGAACGAATT-3', 5' -GCTTTCTCCATGGTGGTGAAGA-3'.

Behavioral analysis. Thermal sensitivity was tested using Hargreaves (radiant heat), hot plate $\left(48^{\circ} \mathrm{C}\right)$, and tail immersion in hot water $(48,50$ and $52^{\circ} \mathrm{C}$ ), and are expressed as paw-withdrawal latency and tail-flick latency The cutoff latency was set to prevent tissue damage (Liu et al., 2012). For testing mechanical sensitivity, the plantar surface of hindpaw was stimulated with a series of von Frey hairs (0.02-2.56 g; Stoelting) and the $50 \%$ paw withdrawal threshold was determined using Dixon's updown method. For assessing formalin-induced acute inflammatory pain, the time (in seconds) that a mouse spent in licking and lifting the affected paw was recorded every $5 \mathrm{~min}$ for $45 \mathrm{~min}$. The experimenters were blinded to the genotypes in all behavioral tests.

Statistical analysis. All data are expressed as mean \pm SEM. Differences between groups were compared using unpaired or paired Student's $t$ test

\section{Increased excitability in DRG neurons of NR1-cKO mice}

Because NR1 is the essential subunit of NMDARs, deletion of NR1 in DRG sensory neurons should render them without functional NMDARs. To test this, we recorded from small-sized sensory neurons using whole-cell voltage-clamp electrophysiology. Bath application of NMDA $(500 \mu \mathrm{M})$ elicited robust inward currents in wild-type but not in NR1-deficient DRG neurons $(0 / 25$; Fig. 2A), confirming the absence of functional NMDARs. Seventy-one percent of wild-type neurons (21/28) expressed functional NMDARs (Fig. 2B).

We next investigated whether deleting NR1 would alter the intrinsic firing properties of DRG neurons under current clamp. NR1-deficient neurons fired more repetitive action potentials after current injection (100 and 250 pA; Fig. 2C,D). However, the resting membrane potential was not altered $(-59.8 \pm 2.4$ vs $-59.6 \pm 2.7 \mathrm{mV}$ for control and NR1-deificient neurons, respectively, $n=9$ neurons/group). The amplitude of action potentials was also not altered $(62.0 \pm 2.3$ vs $59.0 \pm 2.5 \mathrm{mV}$ for control and NR1-deificient neurons, respectively, $n=9$ neurons/group).

To determine whether there is endogenous glutamate release from DRG neuronal cell bodies to cause NMDAR-dependent 
A

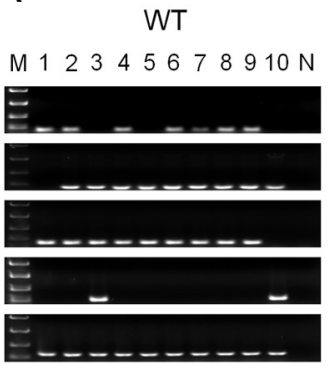

$\mathrm{D}$

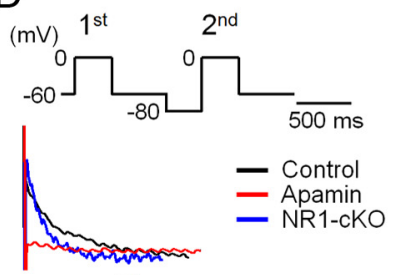

$1^{\text {st }}$

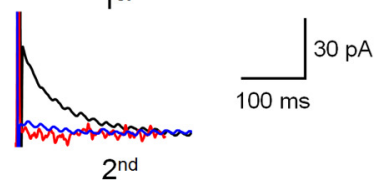

NR1-cKO

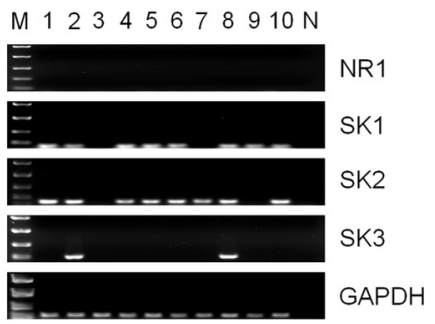

E
B

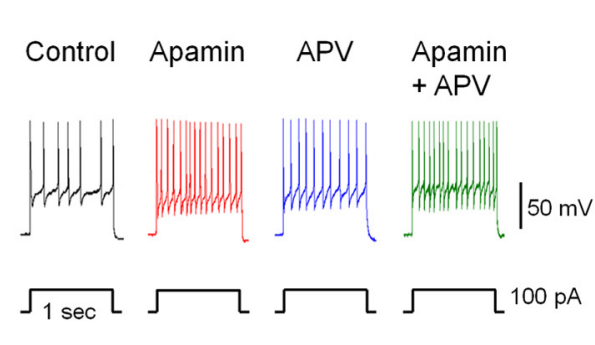

C

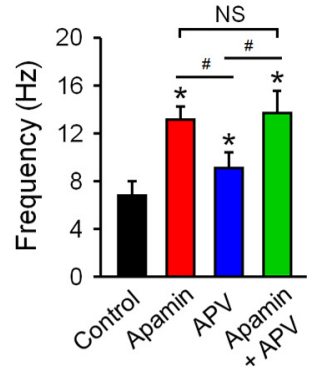

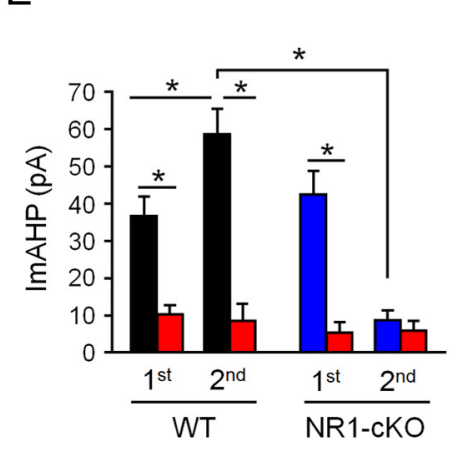

$\mathrm{F}$

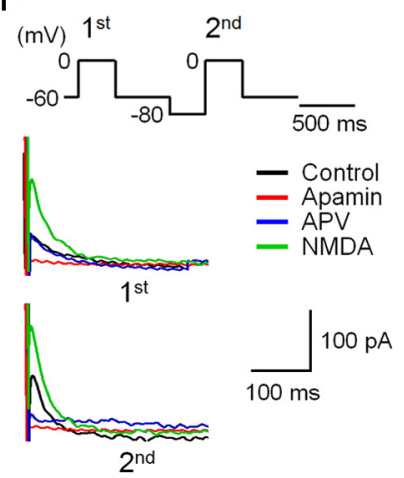

G

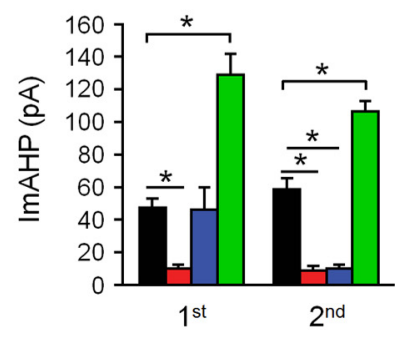

Figure 3. NR1 deficiency and NMDAR blockade cause suppression of apamin-sensitive SK channel activities and hyperexcitability in small-sized DRG neurons. $\boldsymbol{A}$, Single-cell RT-PCR in 10 small-sized DRG neurons showing the expression and colocalization of SK1, SK2, SK3, and NR1. Note that most neurons express NR1 (70\%), SK1 (90\%), and SK2 (90\%), but not SK3 (20\%). GAPDH, positive control; M, molecular weight; N, negative control. B, C, Apamin (100 nM) and APV (50 $\mu$ ) increase action potential frequency in DRG neurons. B, Traces of action potentials. C, Frequency of action potentials. Note that APV plus apamin produces no further increase in frequency compared with apamin alone. ${ }^{*} p<0.05$ compared with control; ${ }^{\#} p<0.05$. N0, no significance, $n=5-6$ neurons/group. D, E, AHP currents are reduced in NR1-deficient neurons after the second pulse stimulation. $\boldsymbol{D}$, Traces of AHP currents in control, apamin-treated, and NR1-deficient DRG neurons. $\boldsymbol{E}$, Amplitude of AHP currents. ${ }^{*} p<0.05, n=7-10$ neurons/group. $F, G$, AHP currents are increased by NMDA and suppressed by APV. $F$, Traces of AHP currents of control and NMDA-, apamin-, and APV-treated DRG neurons. G, Amplitude of AHP currents. ${ }^{*} p<0.05, n=5-12$ neurons/group.

inward current, we used whole-mount DRGs for whole-cell voltage-clamp recordings. Depolarization of DRG neurons by current injection induced marked inward currents in 20 of 38 neurons and APV blocked the inward currents (Fig. 2E,F). The same stimulation failed to induce inward currents in all six NR1deficient neurons we recorded (data not shown). Therefore, depolarization may induce NMDAR-dependent inward currents in DRG cell bodies via endogenous glutamate release.

\section{Reduction in activity but not expression of SK channels in} DRG neurons of NR1-cKO mice

$\mathrm{Ca}^{2+}$-activated SK channels (SK1, SK2, and SK3) are expressed in DRG neurons (Mongan et al., 2005) and play an important role in regulating action potentials (Faber, 2010; Adelman et al., 2012). Activation of NMDARs and voltage-gated $\mathrm{Ca}^{2+}$ channels contributes to the activation of SK channels in cortical neurons (Adelman et al., 2012). Single-cell RT-PCR analysis in small-sized DRG neurons revealed that NR1, SK1, SK2, and SK3 were expressed by $70 \%, 90 \%, 90 \%$, and $20 \%$ of neurons, respectively (Fig. 3A). All NR1-positive neurons expressed SK2 (Fig. 3A), providing a cellular basis for NMDAR modulation of SK activity. As expected, NR1 expression was completely lost in NR1-cKO mice, but SK1/SK2/SK3 expression was unaltered after NR1 deletion (Fig. 3A).

To determine the function of SK in DRG neurons, we selectively blocked SK with apamin (Adelman et al., 2012). Apamin (100 nM) markedly increased action potential frequency (Fig. $3 B, C)$. The NMDAR antagonist APV $(50 \mu \mathrm{M})$ also significantly increased the action potential frequency, although the frequency was lower than that of apamin treatment (Fig. 3B,C). A com- bined treatment of apamin and APV did not further increase the frequency over that of apamin alone, suggesting that APV may modulate action potentials via SK.

We further isolated apamin-sensitive outward AHP currents in DRG neurons as described previously (Gold et al., 1996). AHP currents were largely suppressed by apamin (100 nM; Fig. 3D-G). AHP currents were not affected in the $\mathrm{cKO}$ mice after the first pulse stimulation ( $400 \mathrm{~ms},-60$ to $0 \mathrm{mV}$ and returning to -60 $\mathrm{mV}$ ). Interestingly, the AHP currents after the second pulse (400 $\mathrm{ms},-80$ to $0 \mathrm{mV}$ and returning to $-60 \mathrm{mV}$ ) were abolished in NR1-deficient neurons (Fig. $3 D, E)$. In parallel, APV $(50 \mu \mathrm{M})$ also suppressed the second pulse-evoked currents, whereas NMDA $(100 \mu \mathrm{M})$ increased the currents (Fig. $3 F, G$ ). Therefore, genetic deletion or pharmacological blockade of NMDARs in DRG neurons may result in hyperexcitability via suppressing the SK channel activity.

Increased thermal and mechanical pain sensitivity and spinal cord synaptic transmission in NR1-cKO mice

NR1-cKO mice showed pain hypersensitivity in several behavioral tests. These mice displayed mechanical hypersensitivity, as indicated by lowered paw withdrawal thresholds to von Frey hair stimuli ( $p<0.05, n=17$ mice/group; Fig. $4 A)$. The cKO mice also exhibited thermal hypersensitivity in response to radiant heat in Hargreaves tests ( $p<0.05, n=6$ mice/group) or hot plate tests ( $p<0.05, n=12$ mice/group). Conversely, hot water tail immersion tests did not show any differences between genotypes at $48^{\circ} \mathrm{C}, 50^{\circ} \mathrm{C}$, and $52^{\circ} \mathrm{C}$ (Fig. $4 B$ ). Furthermore, both the first phase $(0-10 \mathrm{~min})$ and the second phase $(10-45 \mathrm{~min})$ responses in the formalin test were unaltered in the NR1-cKO mice (Fig. 
A

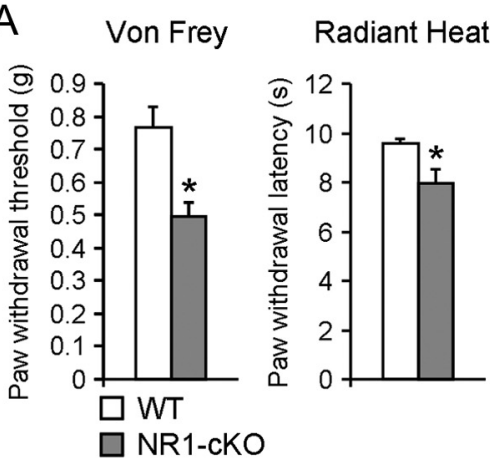

Hot plate B

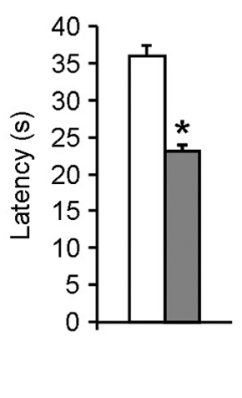

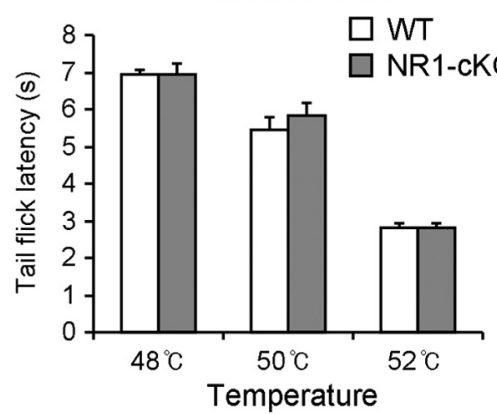

C

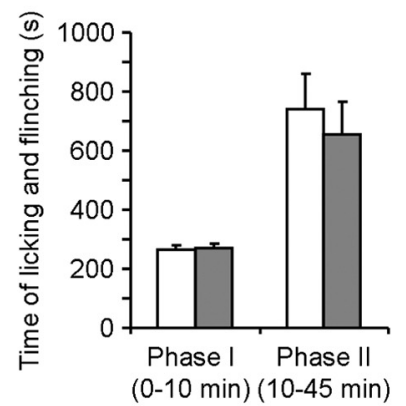

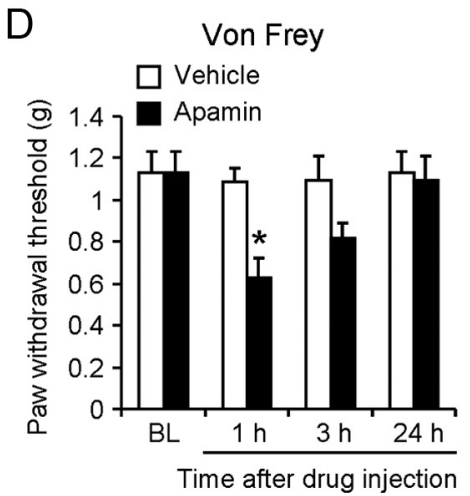

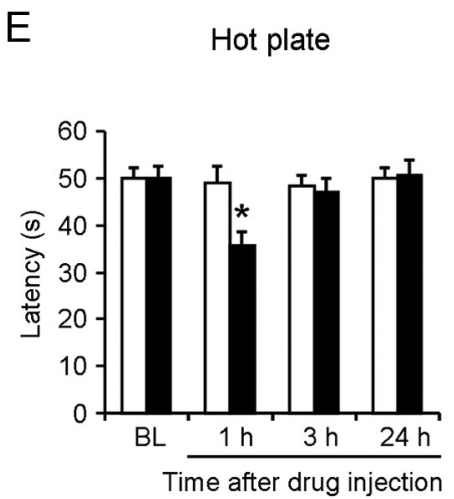

$\mathrm{F}$

G
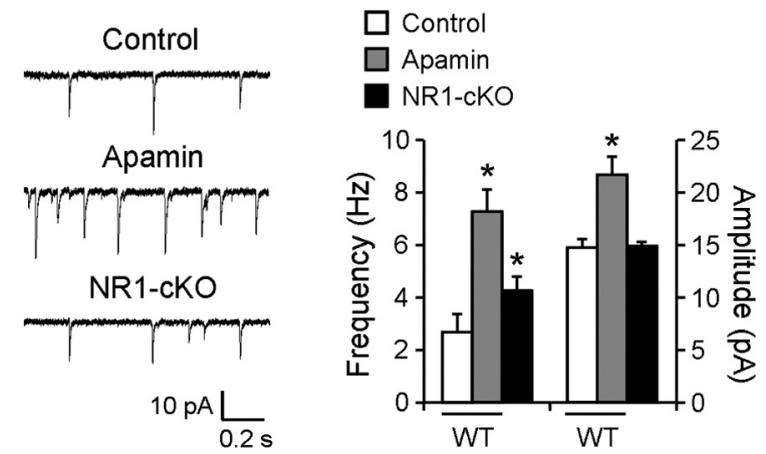

Figure 4. NR1-cKO and apamin-treated mice show pain hypersensitivity and enhanced synaptic transmission. A, NR1-cKO mice display reductions in paw withdrawal threshold in the Von Frey test $\left(n=17\right.$ mice/group), paw withdrawal latency in the Hargreaves test $\left(n=6\right.$ mice/group), and paw withdrawal latency in the hot plate test at $48^{\circ} \mathrm{C}(n=12 \mathrm{mice} / \mathrm{group}) .{ }^{*} p<0.05$ compared with wild-type littermate controls. $\boldsymbol{B}$, Tail immersion test showing unaltered tail flick latency in NR1-cK0 mice. $n=6$ mice/group. C, Formalin test showing normal first phase ( $0-10$ min) and second phase $(10-45 \mathrm{~min})$ pain in cKO mice. $n=6$ mice/group. $\boldsymbol{D}, \boldsymbol{E}$, Intraplantar injection of apamin $(200 \mathrm{ng})$ induces mechanical allodynia (von Frey test, $\boldsymbol{D})$ and heat hyperalgesia (hot plate test, $\boldsymbol{E}){ }^{*} p<$ 0.05 compared with vehicle controls, $n=6$ mice/ group. $F, G, \mathrm{mEPSC}$ in spinal lamina II neurons in spinal cord slices of NR1-cKO mice and wild-type mice before and after apamin (100 nm) treatment. $\boldsymbol{F}$, Representative traces of mEPSCs. G, Frequency and amplitude of mEPSCs. ${ }^{*} p<0.05, n=6$ neurons/group.

4C). The discrepancy in different pain tests may be due to different sensitivity in these tests or to different contributions of peripheral versus central NMDARs to pain sensitivity. In support of electrophysiological results (Fig. 3), intraplantar injection of apamin (200 ng) induced marked mechanical and heat hypersensitivity, which recovered after $3 \mathrm{~h}$ (Fig. $4 D, E$ ).

Finally, we investigated whether presynaptic NR1 and SK are involved in spinal cord synaptic transmission. Patch-clamp recording in outer lamina II neurons, presumably excitatory neurons (Park et al., 2011), showed a significant increase in miniature EPSC (mEPSC) frequency but not amplitude in NR1cKO mice (Fig. $4 F, G$ ), indicating a presynaptic mechanism. Superfusion of spinal cord slices with apamin (100 nM) also increased the frequency and amplitude of mEPSCs (Fig. $4 F, G$ ).

\section{Discussion}

Previous studies implicated conflicting roles of NMDARs in the peripheral and central terminals of primary sensory DRG neurons in pain control (Liu et al., 1997; Coggeshall and Carlton, 1998; Du et al., 2003; Bardoni et al., 2004). Our study demonstrated that peripheral NMDARs control the intrinsic excitability of DRG neurons. Specifically, NR1-deficient DRG neurons exhibited marked increase in action potential frequency. This increase in excitability in NR1-cKO mice can be recapitulated by pharmacological inhibition of NMDARs in wild-type neurons. Consistently, NR1-cKO mice exhibited enhanced mEPSC frequency in lamina IIo neurons due to increased glutamate release from presynaptic terminals. These lamina IIo neurons are predominantly excitatory and receive C-fiber input (Todd, 2010;
Park et al., 2011). It is conceivable that the hyperexcitability in DRG nociceptive neurons and enhanced excitatory synaptic transmission in spinal nociceptive circuit lead to pain hypersensitivity in NR1-cKO mice.

In a previous study, NR1 was deleted in peripherin-expressing DRG neurons (McRoberts et al., 2011), which resulted in an $\sim 75 \%$ reduction of NR1 expression as determined by Western blot analysis. In these peripherin-NR1-cKO mice, no detectable difference in thermal or mechanical sensitivity was observed, but the mice showed a reduction in formalin-induced second phase pain (McRoberts et al., 2011). In this study, we deleted NR1 using Advillin-Cre. Advillin is known to be selectively expressed by all neurons in DRGs (Hasegawa et al., 2007), which is further supported by the observation that NR1 expression was lost in essentially all DRG neurons, but not in central spinal cord neurons of our NR1-cKO mice (Fig. 1A,B). Consistently, NMDA-induced inward currents were completely lost in NR1-deficient DRG neurons (Fig. 2A), whereas the primary afferent projections to the superficial and deep dorsal horn were normal in the mutant mice (Fig. 1C). Unlike peripherin-NR1-cKO, NR1 deletion in Advillin-expressing DRG neurons resulted in pain hypersensitivity in the von Frey (mechanical sensitivity), Hargreaves, and hot plate (heat sensitivity) tests, but normal inflammatory pain in the formalin test. This discrepancy may result from complete deletion of NR1 in all DRG neurons using AdvillinCre versus partial deletion of NR1 in subsets of DRG neurons using peripherin-Cre driver. Furthermore, peripherin is also expressed by motor neurons and other CNS neurons (Allen 
Brain Atlas, http://mouse.brain-map.org/experiment/show/ 70232000), raising the possibility of NR1 deletion in nonDRG neurons using peripherin-Cre.

How are NMDARs in DRG somata activated? Is there activitydependent glutamate release in DRG? Our DRG whole mount recording showed that current injection was sufficient to induce APV-sensitive inward currents in neuronal somata (Fig. 2E,F). Therefore, depolarization may induce NMDAR-dependent inward currents in DRG cell bodies via autocrine or paracrine glutamate release. This result has provided evidence for possible glutamate transmission in the cell body in addition to synapses. Our finding may also suggest a new mechanism for neuronneuron interactions in DRG.

How do NMDARs regulate neuronal excitability in DRG? Our data showed that $\mathrm{Ca}^{2+}$-activated and apamin-sensitive SK in DRG neurons could be involved (Fig. 3D-G). First, SK1 and SK2 are expressed in most small-sized DRG neurons that coexpressed NR1 (Fig. 3A). Second, both APV and apamin increased the number of action potentials in DRG neurons. Third, apaminsensitive tail currents after the second pulse stimulation were lost in NR1-deficient neurons and suppressed by APV in wild-type neurons. Fourth, NMDA increased the apamin-sensitive currents in wild-type DRG neurons. SK channels were originally thought to mediate AHP that follows action potentials. They are also expressed by postsynaptic neurons to regulate synaptic transmission and dendritic excitability (Adelman et al., 2012). Calcium influx through NMDARs, as well as L-type calcium channels, R-type calcium channels, and calcium release from IP3 receptor-sensitive calcium stores, can activate SK (Faber, 2010; Adelman et al., 2012). It is plausible that in DRG neurons, calcium influx through NMDARs is an important source of $\mathrm{Ca}^{2+}$ for SK activation, although we should not exclude the role of other calcium channels. Loss of NMDARs in DRG neurons results in suppressed SK activity, causing these neurons to become hyperexcitable. Consistently, apamin increased excitability in DRG neurons, excitatory synaptic transmission in spinal cord neurons, and pain sensitivity (Figs. 3, 4).

It is likely that peripheral NMDARs may play different roles under different pain or injury conditions. Injury-induced activation of NMDARs in the spinal cord is the best known mechanism for central sensitization. NMDAR-mediated $\mathrm{Ca}^{2+}$ influx activates several protein kinases, such as $\mathrm{CaM}$ kinase, protein kinase $\mathrm{C}$, protein kinase $\mathrm{A}$ (via $\mathrm{Ca}^{2+}$-dependent adenylate cyclase), and MAP kinase for the initiation and maintenance of central sensitization (Ji et al., 2003). However, NMDAR-mediated $\mathrm{Ca}^{2+}$ influx also activates $\mathrm{Ca}^{2+}$-dependent SK channels, serving as a negative feedback to control central sensitization and peripheral sensitization. Our findings call for attention to possible antinociceptive effects of NMDAR activation in primary sensory neurons and also possible opposing effects of NMDAR antagonists for the treatment of chronic pain and other neurological diseases.

\section{References}

Adelman JP, Maylie J, Sah P (2012) Small-conductance $\mathrm{Ca}^{2+}$-activated K+ channels: form and function. Annu Rev Physiol 74:245-269. CrossRef Medline

Aley KO, Levine JD (2002) Different peripheral mechanisms mediate enhanced nociception in metabolic/toxic and traumatic painful peripheral neuropathies in the rat. Neuroscience 111:389-397. CrossRef Medline
Bardoni R, Torsney C, Tong CK, Prandini M, MacDermott AB (2004) Presynaptic NMDA receptors modulate glutamate release from primary sensory neurons in rat spinal cord dorsal horn. J Neurosci 24:2774-2781. CrossRef Medline

Coggeshall RE, Carlton SM (1998) Ultrastructural analysis of NMDA, AMPA, and kainate receptors on unmyelinated and myelinated axons in the periphery. J Comp Neurol 391:78-86. CrossRef Medline

da Silva S, Hasegawa H, Scott A, Zhou X, Wagner AK, Han BX, Wang F (2011) Proper formation of whisker barrelettes requires peripheryderived Smad4-dependent TGF-beta signaling. Proc Natl Acad Sci U S A 108:3395-3400. CrossRef Medline

deGroot J, Zhou S, Carlton SM (2000) Peripheral glutamate release in the hindpaw following low and high intensity sciatic stimulation. Neuroreport 11:497-502. CrossRef Medline

Du J, Zhou S, Coggeshall RE, Carlton SM (2003) N-methyl-D-aspartateinduced excitation and sensitization of normal and inflamed nociceptors. Neuroscience 118:547-562. CrossRef Medline

Faber ES (2010) Functional interplay between NMDA receptors, SK channels and voltage-gated $\mathrm{Ca}^{2+}$ channels regulates synaptic excitability in the medial prefrontal cortex. J Physiol 588:1281-1292. CrossRef Medline

Finch PM, Knudsen L, Drummond PD (2009) Reduction of allodynia in patients with complex regional pain syndrome: A double-blind placebocontrolled trial of topical ketamine. Pain 146:18-25. CrossRef Medline

Gold MS, Shuster MJ, Levine JD (1996) Role of a Ca(2+)-dependent slow afterhyperpolarization in prostaglandin E2-induced sensitization of cultured rat sensory neurons. Neurosci Lett 205:161-164. CrossRef Medline

Hasegawa H, Abbott S, Han BX, Qi Y, Wang F (2007) Analyzing somatosensory axon projections with the sensory neuron-specific Advillin gene. J Neurosci 27:14404-14414. CrossRef Medline

Ji RR, Kohno T, Moore KA, Woolf CJ (2003) Central sensitization and LTP: do pain and memory share similar mechanisms? Trends Neurosci 26: 696-705. CrossRef Medline

Liu H, Mantyh PW, Basbaum AI (1997) NMDA-receptor regulation of substance P release from primary afferent nociceptors. Nature 386:721-724. CrossRef Medline

Liu T, Berta T, Xu ZZ, Park CK, Zhang L, Lü N, Liu Q, Liu Y, Gao YJ, Liu YC, Ma Q, Dong X, Ji RR (2012) TLR3 deficiency impairs spinal cord synaptic transmission, central sensitization, and pruritus in mice. J Clin Invest 122:2195-2207. CrossRef Medline

McHugh TJ, Jones MW, Quinn JJ, Balthasar N, Coppari R, Elmquist JK, Lowell BB, Fanselow MS, Wilson MA, Tonegawa S (2007) Dentate gyrus NMDA receptors mediate rapid pattern separation in the hippocampal network. Science 317:94-99. CrossRef Medline

McRoberts JA, Ennes HS, Marvizón JC, Fanselow MS, Mayer EA, Vissel B (2011) Selective knockdown of NMDA receptors in primary afferent neurons decreases pain during phase 2 of the formalin test. Neuroscience 172:474-482. CrossRef Medline

Mongan LC, Hill MJ, Chen MX, Tate SN, Collins SD, Buckby L, Grubb BD (2005) The distribution of small and intermediate conductance calciumactivated potassium channels in the rat sensory nervous system. Neuroscience 131:161-175. CrossRef Medline

Park CK, Lü N, Xu ZZ, Liu T, Serhan CN, Ji RR (2011) Resolving TRPV1and TNF- $\alpha$-mediated spinal cord synaptic plasticity and inflammatory pain with neuroprotectin D1. J Neurosci 31:15072-15085. CrossRef Medline

Sato K, Kiyama H, Park HT, Tohyama M (1993) AMPA, KA and NMDA receptors are expressed in the rat DRG neurones. Neuroreport 4: 1263-1265. CrossRef Medline

Sawynok J, Reid A (2002) Modulation of formalin-induced behaviors and edema by local and systemic administration of dextromethorphan, memantine and ketamine. Eur J Pharmacol 450:153-162. CrossRef Medline

Todd AJ (2010) Neuronal circuitry for pain processing in the dorsal horn. Nat Rev Neurosci 11:823-836. CrossRef Medline

Zhou X, Wang L, Hasegawa H, Amin P, Han BX, Kaneko S, He Y, Wang F (2010) Deletion of PIK3C3/Vps34 in sensory neurons causes rapid neurodegeneration by disrupting the endosomal but not the autophagic pathway. Proc Natl Acad Sci U S A 107:9424-9429. CrossRef Medline 